\title{
Para entender la paradiplomacia
}

ZIDANE ZERAOUI ${ }^{*}$

En las últimas décadas, el concepto de paradiplomacia se ha hecho presente en las discusiones académicas y en la teoría de las Relaciones Internacionales, desplazando paulatinamente la idea estato-céntrica del sistema internacional.

Si bien este concepto está presente en muchas publicaciones, aún no ha sido plenamente definido. Aunque el análisis del fenómeno inició desde la década de los años setenta, se limitó a una descripción de las actividades internacionales de las regiones, más que a un debate conceptual. Frente a esta carencia, cuando Ivo Duchacek (1984) y Soldatos Panayotis (1988) propusieron el término de paradiplomacia, éste fue rápidamente aceptado, debido a la ausencia de un término más adecuado. Un fenómeno similar se dio con el concepto de Relaciones Internacionales. Cuando Jeremy Bentham acuñó el término en 1780, se refería a relaciones que no eran internacionales, sino entre Estados, pero que no solo se limitaba exclusivamente a las relaciones interestatales (Halliday, 2002). En la actualidad, a pesar de la conciencia de que el término no es el más idóneo, se ha adoptado como el de mayor uso en el análisis internacional.

Así, aun cuando se reconocen los límites del término de paradiplomacia, lo tomaremos como un concepto global para integrar a las demás acepciones, a pesar que desde 1985 algunas voces han criticado la propuesta de Duchacek y Soldatos, como lo ha hecho John Kincaid (citado por Blindenbacher y Koller, 2003). Refiriéndose a la

\footnotetext{
* Profesor-investigador del Instituto Tecnológico y de Estudios Superiores de Monterrey (ITESM). Autor de varios trabajos, entre otros: Los procesos mundiales contemporáneos, (2009); Regionalización y paradiplomacia. La política internacional de las regiones, (2009); Teoría y práctica de la paradiplomacia, (2011) y Islam y política, (2013).
} 
actividad internacional de las regiones, el investigador norteamericano exclama "¿Cómo llamar a esta actividad? Paradiplomacia, pero "para" significa al lado, de un lado, al lado de, o algo secundario. (...) en cambio diplomacia constitutiva toma la idea que los Estados, las provincias, los cantones, los Länders son unidades constitutivas de la política federal" (Kuznetzov, 2015). Aunque la crítica de Kincaid tiene algún fundamento, no logra abarcar a la totalidad del fenómeno internacional de las regiones, como la protodiplomacia, que lejos de ser constitutiva es disruptiva. Sin embargo, no toda actividad internacional de las regiones es "constitutiva" de la política federal y en muchos casos puede ser opuesta; ejemplo de esto es el caso de Cataluña.

Además del aporte de Kincaid, se hace referencia a la diplomacia paralela, a la diplomacia pública, a la diplomacia convergente, a la microdiplomacia, a la protodiplomacia, y a la diplomacia identitaria, entre otras. Nuestra propuesta analítica es utilizar el término de paradiplomacia, con todas sus limitaciones, como un concepto 'paraguas', que incluya a todas las demás acepciones que representan casos específicos de política internacional de las regiones. La diplomacia convergente, por ejemplo, representa una paradiplomacia de la región complementaria de la política nacional, mientras que la protodiplomacia es una paradiplomacia con visos al separatismo.

Por otra parte, planteamos que al instar de la diplomacia, que es exclusiva del poder político federal y no de entidades privadas, la paradiplomacia es una actividad reservada a los gobiernos intermedios, ${ }^{1}$ provincias o municipalidades y no a las instituciones privadas. Las universidades o las empresas privadas pueden tener actividades internacionales o relaciones internacionales, pero diferenciadas de la diplomacia o la paradiplomacia.

Para Cornago (2000), la paradiplomacia es "la participación de gobiernos no centrales en las relaciones internacionales a través del

\footnotetext{
1 Hablamos de una manera genérica de provincias, aunque en la práctica los términos referentes a los gobiernos intermedios varían de país a país: autonomías para España, estados para México, provincias para Argentina, departamento o región en Francia, länders en Alemania, intendencias en Chile, etc.
} 
establecimiento de contactos ad hoc con entidades privadas o públicas del extranjero, con el fin de promover asuntos socioeconómicos y culturales, así como cualquier otra dimensión externa de sus competencias constitucionales"(p. 66). En su definición, este autor enfatiza el papel de las entidades "no centrales", es decir, de los gobiernos intermedios, y no de las empresas privadas o de las instituciones no gubernamentales. En esta misma línea encontramos también a Duchacek que precisa que son "las relaciones de los gobiernos no centrales con centros culturales, comerciales e industriales de otros Estados incluyendo relaciones con los gobiernos de Estados extranjeros. Y se distingue de la diplomacia normal en que es específica sectorialmente, más que en representar a la unidad territorial como un todo" (Iglesias y Zubelzú, 2008). El padre de la paradiplomacia en las Relaciones Internacionales se enfoca en la problemática de Quebec, cuando menciona la relación con "los gobiernos extranjeros", característica muy peculiar de la provincia francesa de Canadá, que se ha distinguido por su grado avanzado de paradiplomacia, incluyendo la apertura de oficinas en el extranjero.

Nuestra posición converge con estos autores en la medida en que reservamos el término de paradiplomacia a los actores gubernamentales, difiriendo en esto de aquellos que atribuyen esta prerrogativa incluso a grupos culturales o nacionales. Por ejemplo, Martins Senhoras (2009) incluye a todos los actores que tienen una actividad internacional, siendo o no estatales; así, en su particular concepción, las empresas o las universidades pueden hacer paradiplomacia.

Tampoco nos situamos en la posición de los autores que ven a la paradiplomacia solamente como una actividad complementaria de la diplomacia tradicional, como lo define Hocking (2000) en su diplomacia multiniveles o a la diplomacia constitutiva de Kincaid, que no ve una acción autónoma de las entidades subnacionales, sino una política de sumisión al poder central. Sin bien es cierto que los gobiernos sub-nacionales generalmente se alinean con el gobierno central en una diplomacia de múltiples niveles o constitutiva, no siempre la política internacional del actor local es un reflejo a pequeña escala de la política nacional. Puede haber intereses divergentes en juego que 
se reflejan particularmente en las entidades fronterizas que defienden sus propias visiones.

La profusión de los conceptos en relación a la actividad internacional de las regiones se debe a la complejidad misma de las entidades y a la variedad de sus actividades internacionales.

Así, para este ensayo vamos a definir, en primer lugar, la paradiplomacia, con el objetivo de avanzar sobre un terreno más despejado conceptualmente. El siguiente apartado se centra en el análisis del origen del concepto, desde su uso en el Derecho Internacional hasta su aceptación en las Relaciones Internacionales. En el campo de las Relaciones Internacionales, hemos utilizado de una manera laxa el concepto de paradiplomacia, mientras el Derecho Internacional define rigurosamente los conceptos de Política Exterior y quien tiene el privilegio exclusivo de esta facultad además del propio concepto de paradiplomacia. Sin embargo, en el Derecho Internacional este término tuvo como origen una misión ad hoc de representantes de un Estado para una finalidad concreta, pero que no son diplomáticos.

En el cuarto apartado incluimos el concepto de marca territorial o marca-región, elemento estrechamente vinculado con la paradiplomacia turística, cultural o comercial. En efecto, para lograr una paradiplomacia exitosa, los gobiernos sub-nacionales deben consolidar su imagen internacional, es decir, deben generar una marca territorial que les permita promover su producción local; de esta manera el concepto de región está íntimamente vinculado al de paradiplomacia. En su obra Kuznetzov (2015) dedica su segundo capítulo a definir el término de región por su relevancia en el debate internacional. Para la Asamblea de las Regiones Europeas, la región es "una unidad territorial inmediatamente debajo del Estado soberano, con un sistema de auto-gobierno, con una propia constitución, un estatuto de autonomía o con cualquier ley que forma parte de la legislación del Estado y que determina como la región es organizada y que poderes tiene" (Sodupe, 1991, p. 59), que le permite precisamente utilizar su propia marca para trascender las fronteras. 
Finalmente, cerramos este trabajo con una reflexión breve sobre los límites de la paradiplomacia y en particular su ausencia de institucionalidad.

\section{Definiendo la paradiplomacia}

La participación de nuevos actores no estatales en la arena internacional (Arts, Noortmann y Reinalda, 2001), ha relegado la preeminencia del Estado central en el sistema global. Sin embargo, para ir definiendo el término debemos limitar el alcance de los conceptos. De una manera general, podemos hablar de paradiplomacia positiva cuando la actividad de la región es complementaria de la nacional o no entra en contradicción con los intereses globales del país. En cambio, la paradiplomacia negativa se refiere al choque de las dos políticas por motivos económicos, políticos o culturales. Por otra parte, no haremos referencia a los casos específicos de la actividad internacional, como en el caso del medioambiente, la cultura y la frontera, que conllevan al uso de los términos de paradiplomacia medioambiental, paradiplomacia cultural y paradiplomacia transfronteriza. No se trata de nuevos conceptos, sino de campos específicos de la aplicación de la paradiplomacia.

Por su parte, Duchacek hace una clasificación entre la paradiplomacia directa e indirecta. "La paradiplomacia directa se da cuando la región o subgrupo tiene una actuación directa en la escena internacional y tiene las verdaderas relaciones al exterior. En cambio la paradiplomacia indirecta, se usa cuando desde el interior se hace presión para moldear las políticas que usara el gobierno central al exterior" (2009, p. 20). En su definición, este autor separa la acción directa de las entidades intermedias en el sistema internacional y las acciones indirectas a través de los grupos de presión o de interés.

Una vez definidas estas acepciones que posicionan a la paradiplomacia en relación al Estado central vamos a analizar las distintas acepciones utilizadas en el medio académico. 
La paradiplomacia convergente o constitutiva hace referencia a una política internacional complementaria entre el Estado central y los gobiernos intermedios. ${ }^{2}$ En este sentido se trata de actividades de apoyo mutuo en donde el Estado central se ve relevado de ciertas tareas puntuales que se delegan a las entidades sub-nacionales; en este caso no hay confrontación entre los intereses de las partes. Por su parte, la diplomacia divergente hace referencia a intereses opuestos entre el poder central y las autoridades locales. En muchos casos la visión del interés nacional puede chocar con las problemáticas regionales; sin embargo, estas divergencias son temporales y no reflejan una oposición permanente entre los intereses de las dos partes.

Cuando una región busca consolidar su imagen internacional, hablamos de paradiplomacia identitaria. La unidad sub-nacional quiere proyectar una cierta marca territorial o ciertos rasgos particulares del territorio. El reforzamiento identitario puede tener su origen en el desarrollo de una imagen regional para promoción del territorio o simplemente como una táctica para posibiltar el rescate de los valores locales. La marca regional está vinculada con esta paradiplomacia, que no solamente tiene un valor simbólico, sino que puede representar una marca propia para promover turísticamente a la región o bien generar una marca de productos locales. Por ejemplo, en el Estado de Chiapas, en México, se ha promovido la "marca Chiapas" para impulsar el desarrollo local sobre una identidad de productos.

Sin embargo, cuando este impulso se lleva a sus extremas consecuencias, es decir, a la imposición de una identidad regional como paso previo a la ruptura con el Estado nacional, estamos frente a una protodiplomacia. La línea entre la diplomacia identitaria y la protodiplomacia es muy delgada, y depende más de la voluntad política de la entidad y su objetivo final. Muchas regiones aun no independientes, han estado enfatizando sus propios valores en detrimento de la

\footnotetext{
2 Retomamos el concepto de gobierno intermedio que es utilizado por la Asociación Latinoamericana de Gobiernos Intermedios (OLAGI), que reúne tanto a gobiernos municipales o como provinciales.
} 
identidad nacional, como podría ser el caso de Cataluña, Flandes, Escocia o el Kurdistán.

\section{El origen de un concepto}

Tanto en los países centralizados, como en las naciones federales, hay presiones contradictorias sobre la conducción de la política exterior: por un lado, existen fuerzas centralizadoras, que buscan lograr una sola voz en el escenario mundial, pero al mismo tiempo hay presiones descentralizadoras, que permiten a los actores subnacionales irrumpir en el sistema internacional.

Estas actividades internacionales de las sub-regiones o de actores no-estatales se han denominado como paradiplomacia. Aunque el concepto ya existía en el derecho internacional desde la década de los años sesenta, en las relaciones internacionales debemos esperar hasta finales de los años setenta para que el término entre en el análisis del sistema mundial y de sus interacciones. "En efecto, la paradiplomacia, o diplomacia paralela que emprenden actores subnacionales, puede leerse desde la década del 80 desde el debate transnacionalista y federalista. Éste da cuenta de la profundización de las actividades internacionales emprendidas fundamentalmente por Gobiernos No Centrales en el contexto de la creciente globalización e interdependencia de las sociedades a distinta escala de acción, posibilitada por los ajustes institucionales del sistema político norteamericano promovidos por el nuevo federalismo, sobre todo en el ámbito de la política exterior y la intromisión de los Estados federados en esta actividad reservada solo para el Estado federal" (Ovando Santana, 2013, p. 2).

Para Aguirre, "existen dos aproximaciones teóricas más que abordan el fenómeno paradiplomático. Uno, el debate promovido por las teorías reflectivistas posmodernas encabezadas por Der Derian (1987) que plantean, escudriñan e interpretan las actividades paradiplomáticas que han emprendido históricamente distintos actores sean o no estatales alojados en la escala regional o subnacional, aunque no admitidos por la diplomacia tradicional estadocentrica centralizada. Y una segunda, a partir de la década del 90, denominada gobernanza multinivel 
(Marks), que responde a los retos institucionales de la ampliación de la Unión europea, sobre todo en lo referido al surgimiento de nuevas instituciones que promueven el desarrollo de las regiones europeas y sus conexiones con otras escalas de acción internacional” (p. 209).

La hegemonía estatocéntrica en la teoría de las relaciones internacionales no había facilitado el interés académico sobre la participación de las regiones en la política internacional, a pesar del surgimiento de los enfoques behavioristas o las teorías parciales, que se centraban en los elementos del sistema internacionales.

A pesar de la ausencia de estudios sobre el fenómeno paradiplomático hasta finales de la década de los años setentasa, éste "no es una novedad. (...) Parece que se puso de moda abrir oficinas en el extranjero, inmiscuirse en los organismos internacionales o estar presente sin descanso en las conferencias. Esta actividad logró frutos como la introducción en la Convención de Lomé IV (de la Unión Europea con los países ACP-África, Caribe y Pacífico) del concepto de cooperación descentralizada en la UE, lo que permitió a organizaciones ajenas al gobierno central, utilizar recursos destinados por la Comisión (europea) a la cooperación" (Mateos, 2010, [en línea]). En Europa existen 250 regiones que acaparan el tercio del presupuesto comunitario, es decir, 213 mil millones de euros para proyectar su imagen a nivel internacional para fines culturales, sociales, económicos o políticos.

La idea de convertir a las regiones en el nivel principal de gobierno conoció su auge en la Unión Europea en la década de los años ochenta, cuando varios países promovieron la regionalización de su política interna, como España, y que la política regional europea estaba en plena expansión. Es en este contexto que los Länder ${ }^{3}$ alemanes lograban la creación del Comité de Regiones en el seno de

\footnotetext{
3 Alemania es una federación de 17 Länder (de Land 'país' o 'estado'). Los Länder son sujetos de derecho internacional, con personalidad estatal propia, aunque la función diplomática la representa el Estado Federal, pero varios Länder tienen acuerdos internacionales y oficinas en las principales ciudades europeas.
} 
la UE. Además, desde el Tratado de Maastricht, ${ }^{4}$ los representantes de los organismos regionales o sub-estatales podían participar en las delegaciones nacionales cuando se negociaba en el seno del Consejo de la Unión. A partir de ahí, representantes de las comunidades y regiones belgas, del gobierno escocés, galés o de Irlanda del Norte, las autonomías españolas, de los Länder alemanes, los austriacos y de las regiones autónomas portuguesas pueden participar en las sesiones del Consejo de Ministros de la Unión europea.

A pesar de su práctica cotidiana, la paradiplomacia sigue siendo un fenómeno poco estudiado. La mayoría de las obras se enfocan en describir el proceso, más no en explicarlo, menos aún, en buscar un fundamento conceptual. "La paradiplomacia sigue siendo desconocida, pero el fenómeno que representa se volvió común: la ciudad de San Francisco sanciona un país extranjero que no respeta los derechos humanos; el gobierno de Quebec inaugura una temporada cultural en París; las entidades subestatales flamencas y valonas conforman la delegación belga en la Organización del Comercio Mundial; los Estados australianos asisten en el seno de la representación gubernamental de Australia en una conferencia de las Naciones Unidas sobre desarrollo y medio ambiente; el Länd de Baden-Wurtemburg participa a las misiones de restablecimiento de la paz en Bengladesh, en Rusia, en Bosnia-Herzegovina, en Burundi y en Tanzania; Jordi Pujol, presidente de Cataluña, se encuentra con el presidente George Bush (padre)" (Paquin, 2003, p.640).

Por otra parte, la paradiplomacia, fenómeno global y extenso en todo el planeta por su carácter poco mediatizado, confuso y complejo, tiene un perfil bajo en los medios de comunicación de masas, aunque en los centros universitarios se ha convertido en un tema de moda.

Las administraciones locales, municipales y estatales pueden promocionarse a nivel regional e internacional, es decir, pueden llevar

\footnotetext{
4 El Tratado de Maastricht de 1992 fue decisivo para el viejo continente, en la medida en que introdujo el mercado común, cambió el nombre de Comunidad Económica Europea a Unión Europea, y finalmente decidió la introducción del Euro como moneda comunitaria.
} 
a cabo acciones acciones externas y de relaciones internacionales que salen del marco centralizado del Estado, tomando decisiones e iniciativas propias. De esta manera, la esencia de la paradiplomacia es "Ela implicación de los Gobiernos No Centrales en las relaciones internacionales, a través del establecimiento de contactos formales e informales, permanentes o ad hoc con entidades extranjeras, públicas o privadas, con el propósito de promover asuntos de carácter socioeconómicos, políticos o culturales, así como cualquier otra dimensión externa de sus competencias constitucionales" (Cornago, 2005, p.8).

Sin embargo, la primacía de la política exterior central del Estado no está en cuestión, a pesar de la creciente relevancia de la política internacional de las regiones. Las prerrogativas del Estado Federal y la concentración de varios poderes en su seno, le permiten seguir siendo el centro de la actividad internacional de los países, aunque hay nuevos patrones en el orden internacional y uno de ellos ha sido la creciente participación de otros actores que no son el Estado tradicional, además de que con la continua apertura democrática y el crecimiento de las comunicaciones, cada vez es más común que las administraciones locales, e incluso empresas e instituciones, tomen iniciativas propias al relacionarse con el exterior.

Las acciones de paradiplomacia son muy variadas. A nivel gubernamental pueden ir desde la administración más básica y local, hasta un conjunto de estados o provincias de un país actuando bajo un mismo propósito, quienes "buscan la vinculación externa, pues se presume que ésta contribuye al mejoramiento de las capacidades institucionales para atraer los recursos y el talento necesarios para gestar su propio desarrollo" (Carreón, 2007, [en línea]).

El término de paradiplomacia, recién en el discurso de los internacionalistas, ${ }^{5}$ es un concepto bastante antiguo en el Derecho

\footnotetext{
5 En efecto, en toda la literatura relacionada a esta problemática, los autores se refieren a Ivo Duchacek y Panayotis Soldatos como los 'padres' de la paradiplomacia. En parte esta aseveración es cierta en la medida que los dos especialistas del Québec, Canadá, fueron los pioneros en introducir este concepto en el campo de las Relaciones Internacionales como
} 
Internacional. Sin embargo, para este último, "la paradiplomacia se integra de grupos muy variados, tal como el de los funcionarios de organizaciones internacionales, los delegados a conferencias internacionales, los agentes ad-hoc, los expertos, los especialistas, los enviados, etcétera, y está exigiendo nuevos tratamientos y reglamentaciones, pues el número de estos agentes y la diversidad de sus ocupantes requieren normas diferentes de las usuales. La intervención masiva de técnicos en las relaciones exteriores de los Estados trae consigo la necesidad de prever alguna competente ordenación legal de sus actividades" (Sepúlveda, 1988, p. 162).

Así, el Derecho Internacional incluye en esta actividad internacional a representantes oficiales de los Estados solamente con una función ad hoc, no a entes subnacionales que actúan por su cuenta o a organismos descentralizados, y menos aún a las empresas o las instituciones educativas. De esta manera, ciertas prácticas paradiplomáticas ya han sido reguladas, en particular las referentes a las actividades de los funcionarios de organizaciones internacionales, sobre todo en relación con su inmunidad en sus relaciones con otros Estados. En cambio, los delegados a conferencias internacionales, los representantes de los gobiernos, los enviados itinerantes y las misiones especiales (que no sean diplomáticos) tienen un tratamiento menor que los anteriores, pero gozan de ciertos privilegios 'diplomáticos'.

lo demuestra la gran cantidad de obras publicadas: Duchacek, Ivo "Perforated Sovereignties: Towards a Typology of New Actors in International Relations" o Soldatos, Panayotis "An Explanatory Framework for the Study of Federated States as Foreign-policy Actors" ambos en Michelmann, H. y Soldatos, P., Federalism and International Relations. The Role of Subnational Units, Oxford, Clarendon Press, 1990; Duchacek, I., Latouche, D., Stevenson, G. (eds.) Perforated Sovereignties and International Relations: Trans-Sovereign Contacts of Subnational Governments, Nueva York, Greenwood Press, 1988; Duchacek, Ivo. The territorial dimension of politics: within, among and across nations, New York, Greenwood Press, 1986 y Soldatos, Panayotis "Cascading Subnational Paradiplomacy in an Interdependent and Transnational World" en Brown, D., Fry, E., States and Provinces in the International Political Economy, Berkeley, California, Institute of Governmental Studies (IGS), 1993. Sin embargo, como lo hemos visto es en realidad en el Derecho Internacional que se empezó a utilizar este término, aunque con referencia a 'actores' designados por los Estados, no independientes. 
Esta necesaria regulación de las actividades paradiplomáticas tiene su origen en la formulación misma del Derecho Internacional. En sus inicios, éste se enfocaba exclusivamente en el Estado, que era considerado como el único agente del Derecho Internacional. Hasta el día de hoy, aunque se acepta que los actores internacionales se han multiplicado, los únicos 'órganos de las relaciones internacionales' se centran en el Estado, como 'órganos centrales' (jefe de Estado y ministerios de Relaciones Exteriores) y 'órganos exteriores' (agentes diplomáticos y consulares). Toda la literatura del Derecho gira en regular y explicar las obligaciones y privilegios de estos órganos, no de los actores paradiplomáticos.

"El sistema internacional, o la sociedad internacional, si se prefiere denominarlo así, se basa fundamentalmente en los Estados como sujetos o actores predominantes. Durante mucho tiempo se sostuvo uniformemente que los Estados eran los únicos sujetos o los sujetos exclusivos del orden jurídico internacional. ${ }^{6}$ Sólo hasta los años veintes empezó a abrirse paso débilmente la tesis de que además del Estado existen otros sujetos $-\mathrm{O}$ actores- titulares de derechos y obligaciones internacionales, y que hay una parte del derecho de gentes, cada vez más creciente, que se dirige a ellos. Esta postura no ha dejado de te-

6 Desde su creación, el Derecho Internacional se concibió para regular las relaciones entre los 'sujetos del Derecho', los Estados, considerados depositarios de los plenos poderes en el ámbito internacional. En el nacimiento del Derecho Internacional, la Universidad de Salamanca, España, fue pionera por los trabajos de los religiosos. El fraile dominico español, Francisco de Vitoria (1483/86-1546), fue el primero en desarrollar una teoría sobre el derecho de gentes (ius gentium) que sin lugar a dudas puede calificarse de moderna. Extrapoló sus ideas de un poder soberano legítimo sobre la sociedad al ámbito internacional, concluyendo que este ámbito también debe regirse por unas normas justas y respetuosas con los derechos de todos. El bien común del orbe es de categoría superior al bien de cada Estado. Esto significó que las relaciones entre estados debían pasar de estar justificadas por la fuerza a estar justificadas por el derecho y la justicia. Así, Francisco de Vitoria se convirtió en el creador del derecho internacional moderno. El ius gentium se fue diversificando con los aportes de otros pensadores de la Universidad de Salamanca. El padre jesuita Francisco Suárez (1548-1617), que ya trabajaba con categorías similares, distinguía entre ius inter gentes e ius intra gentes. Mientras que el ius inter gentes, que correspondería al derecho internacional moderno, era común a la mayoría de países (por ser un derecho positivo, no natural, no tiene porqué ser obligatorio a todos los pueblos), el ius intra gentes o derecho civil, es específico de cada nación. 
ner oposición, sobre todo de los positivistas, que mantienen que el único derecho internacional es aquél producido por la 'voluntad del Estado', y que lo demás es sólo una emanación subsidiaria” (Sepúlveda, 1988, p. 476).

En efecto, el derecho internacional se preocupa primordialmente por los Estados, que son los que definen y crean el derecho, aunque su aplicación puede abarcar a otros actores, pero secundarios en términos de la jurisprudencia del sistema mundial. "La concepción y la definición del derecho internacional, tal como han sido propuestas por los escritores en cualquier periodo de la historia, han ejercido una profunda influencia en el problema de determinar quiénes son los sujetos de derecho internacional. Así, de acuerdo con la definición clásica que considera el derecho internacional como un conjunto de normas que rigen la conducta de los Estados, en sus relaciones mutuas, sólo éstos, los Estados, son sujetos de derecho internacional" (Sorensen, 2000, p. 261). Aunque el derecho internacional actual se preocupa por el papel de los individuos y de las instituciones internacionales, y busca otorgarles derechos pero también responsabilidades, es un hecho que su propia elaboración sigue siendo una facultad casi exclusiva de los Estados.

El derecho internacional reconoce la existencia de otros sujetos, además de los Estados soberanos y las instituciones internacionales (que son una expresión del rol del Estado central), pero con un rango menor. Así "hay otros sujetos menos importantes de derecho internacional que, sin embargo, ocupan una posición especial en el orden jurídico internacional. Si alguna característica común puede señalárseles, es que todos carecen de uno u otro de los requisitos esenciales de un Estados soberano. Es decir carecen, ya sea de autoridad gubernamental, de población, de territorio o de soberanía y, especialmente, de soberanía en sus relaciones exteriores. En este grupo de sujetos de derecho internacional, consideramos entidades tales como la Santa Sede, los Estados diminutos, las colonias, los protectorados, algunos territorios autónomos, los territorios en fideicomiso, las partes beligerantes y los insurgentes" (Sorensen, 2000, p. 271). 
Todos los actores señalados por Sorensen son 'casi-Estados' o 'paraEstados', pero no subregiones, a menos que entre en conflicto bélico con el poder central, o entidades no públicas, es decir, instituciones académicas, empresas transnacionales, etc.

El derecho internacional funciona dentro de la comunidad de los Estados soberanos, cuyas políticas exteriores conforman sus relaciones, y esto se pone en ejecución a través de la diplomacia. Estas consideraciones conllevan a establecer que solamente las entidades soberanas pueden ejercer una diplomacia y una política exterior, mientras que los actores no-estatales realizan paradiplomacia y política internacional.

De esta manera, "la política exterior es el conjunto de decisiones tomadas por un gobierno en relación con la posición del Estado vis-à-vis otros Estados y, además, su actitud dentro de las organizaciones y conferencias internacionales. La diplomacia es el instrumento mediante el cual se lleva a efecto la política exterior" (Sorensen, 2000, p. 385). Así, queda muy clara la diferencia en el actuar en el escenario mundial entre el Estado y los demás actores con proyección internacional.

Seara Vázquez (1988) reconoce que "el estudio del Estado corresponde fundamentalmente al derecho político y constitucional (...) por ser el sujeto principal del derecho internacional" (p. 83). Las regiones se convierten en sujeto del derecho internacional solamente en estado de beligerancia y no por sus acciones en el sistema mundial. Por los reconocimientos 'de beligerancia, de insurgencia y del derecho a la independencia', otras entidades se convierten en sujetos de derecho.

Esta posición limitada del derecho internacional se encuentra también en la visión del realismo político, ${ }^{7}$ dominante durante toda la Guerra Fría, que sostiene que los únicos actores internacionales son

\footnotetext{
7 Cfr. a los clásicos del realismo político Morgenthau, Hans Joachim. Politics among nations: the struggle for power and peace, Nueva York, Knopf Editores, 1985 (la primera edición es de 1948) y Waltz, Kenneth Neal. Man, the state and war: a theoretical analysis, Nueva York, Columbia Press, 2001 (primera edición de 1954). Sin embargo, el realismo político sigue siendo un referente central de la idea del Estado como actor único de las Relaciones Internacionales: Pont Mestres, Magín. Realismo politico, Madrid, Plaza \& Janes, 1977; Bethke Elshtain, Jean.
} 
los Estados y que los demás entes son subordinados a la voluntad del poder estatal o que, en el mejor de los casos, expresan, de manera distinta, esta misma voluntad.

\section{Paradiplomacia y marca territorial}

Un elemento central en la paradiplomacia es la identidad regional; para que una provincia pueda tener un rol regional o internacional debe consolidar su imagen. Según Abderrahmane Rachik (1995) existen dos identidades: la dura y la blanda. La primera se refiere primordialmente a los irredentismos que se oponen abiertamente a la cultura nacional, mientras que la identidad blanda existe en todas las provincias o regiones y es lo que permite diferenciar un Estado de otro, sin hacer de estas diferencias antagonismos.

Relacionado con este elemento, las identidades pueden ser inclusivas o exclusivas, tal como lo definen Klotz y Lynch (2007). Generalmente las identidades duras suelen ser exclusivas, mientras que las identidades blandas tienen tendencia a ser más inclusivas, pero no es una regla permanente.

Por su parte, la identidad debe ser territorializada para poder expresarse. En efecto, sin la territorialización de la identidad, ésta no puede existir. Sin embargo, debemos diferenciar el espacio del territorio. Así, el primero se refiere a una extensión geográfica que no necesariamente genera un vínculo afectivo con la población, mientras que el territorio es un espacio valorizado (Giménez y Malgesini, 2000). De esta manera, la territorialidad puede definirse como la capacidad humana de producir "territorios" mediante la delimitación y la valorización espacial, lo que permite consolidar la identidad, base de toda actividad paradiplomática. Desde el momento de delimitación y valorización, se desarrolla un apego por este espacio que provee de recursos a sus habitantes.

Real Politics: at the center of everyday life, Baltimore, John Hopkins Press, 2000; Hasan Siddiqi, Safdar. Real politics: a new approach, Karachi, Pakistán, Ferozsons, 2003. 
Sin embargo, la existencia de una identidad regional no implica la negación de la identidad nacional. Podemos tener varias identidades superpuestas que se complementan unas a otras; de hecho, varias micro-identidades territoriales pueden compartir una sola macroidentidad territorial.

El estudio de la paradiplomacia quedaría incompleto sin la inclusión de las identidades y dela marca territorial que le den una autonomía propia. Precisamente, la marca región se ha analizado no solamente en el marco de las relaciones internacionales, sino en varios campos disciplinarios, como la política, la cultura, el comercio, el turístico, el desarrollista y el comunicacional.

"La política económica y comercial, la promoción de las inversiones extranjeras y la atracción de centros de decisiones, la promoción de las exportaciones, la ciencia y la tecnología, la energía, el medio ambiente, la educación, la inmigración y la movilidad de las personas, las relaciones multilaterales, le desarrollo internacional y los Derechos Humanos forman los principales expedientes de la paradiplomacia" (Paquin, 2004, p. 207).

De esta manera, el estudio de la identidad territorial es inherente al estudio de las regiones y de su necesidad de proyectarse hacia el exterior como entidad autónoma, con intereses definidos y claramente separados tanto de los del Estado central como de las demásregiones.

\section{Conclusión: Los límites de la paradiplomacia}

A diferencia de la diplomacia tradicional, que está institucionalizada en la figura de las secretarías de relaciones exteriores o los ministerios de asuntos internacionales, la paradiplomacia está íntimamente ligada a la figura del líder político regional. En el Estado de Nuevo León, en México, durante su gubernatura (2004-2010), Natividad González Parás impulsó a Monterrey como Ciudad del Conocimiento y como centro médico alternativo a Houston; además, con su programa "Invite" vinculó a Nuevo León con Tamaulipas, Coahuila y el Estado de 
Texas (la vieja relación colonial) para generar un bloque negociador a nivel global.

Su sucesor, Medina, frente a la crisis de seguridad en el Estado (y a nivel nacional durante los años 2010-2012) se centró en la problemática interna, dejando de lado la política internacional de Nuevo León. La ausencia de una institucionalización de las actividades paradiplomáticas conlleva a su debilidad y su vinculación con el liderazgo en turno.

Por otra parte, tampoco existe una definición constitucional de la actividad paradiplomática. Durante la Guerra Fría, los Estados centrales no permitían una autonomía de los gobiernos intermedios a nombre de la seguridad nacional del Estado. Con el derrumbe del mundo bipolar, en varios países la necesidad de regular las actividades internacionales de las provincias que se dieron por el propio debilitamiento del Estado nacional, se llevaron a cabo reformas constitucionales.

La revisión Constitucional en México en el año 1992 permitió abiertamente la cooperación exterior de los Estados en el marco de la política internacional del poder central. Sin embargo, esta reforma no precisa los alcances de la paradiplomacia y se limita a señalar que los Estados deben "notificar" a la Secretaría de Relaciones Exteriores de cualquier acuerdo firmado, pero no existen sanciones en caso de incumplimiento.

Por su parte, en Argentina la reforma constitucional de 1994, abrió la puerta a la legitimización de la política internacional de las provincias o municipios. El artículo 124 de la Carta Magna permite su proyección internacional y afirma la facultad de celebrar tratados internacionales, conforme los límites que establece el propio artículo 124 para las provincias. Además, reconoce a las regiones como nuevos sujetos del derecho internacional, legitimadas para actuar en el escenario mundial, al igual que las provincias. La Ciudad Autónoma de Buenos Aires tiene la legitimidad para celebrar convenios internacionales conforme lo establece la Constitución Nacional, como también lo dispone su estatuto organizativo. 
Así, aunque varios países hayan incluido en su Constitución la actividad internacional de sus provincias, no está claro los límites de los gobiernos intermedios.

Para los Estados centrales, y varios autores comparten esta visión, la paradiplomacia, retomando la definición de Paquin (2004) es "un mandato dado a representantes oficiales por un gobierno sub-estatal para negociar con actores internacionales" (p. 209), pero los límites de la paradiplomacia depende de las correlación de poder entre la región y el centro. Es decir, el alcance de la actividad internacional de los gobiernos intermedios depende de su propia voluntad y de su capacidad de negociar con el poder central.

\section{Referencias}

Aldecoa, F., \& Keating, M. (2000). Paradiplomacia: Las relaciones internacionales de las regiones. Madrid: Editorial Marcial Pons.

Arts, B., Noortmann, M., \& Reinalda, B. (2001). Non-State Actors in International Relations. Aldershot: Ashgate.

Bethke Elshtain, J. (2000). Real Politics: at the center of everyday life. Baltimore: John Hopkins Press.

Carreón, P. (2007). Paradiplomacia y su desarrollo en el mundo. Recuperado de: http://www.protocolo.com.mx/articulos.php?id_sec=3\&id_ art $=2548 \&$ \&id_ejemplar $=0$

Cornago, N. (2001). Exploring the global dimensions of paradiplomacy: Functional and normative dynamics in the global spreading of subnational involvement in foreign affairs. Ponencia presentada en Forum of Federations/Forum des Fédérations; Ottawa; http://www.ciff.on.ca (acc. 20/06/02) citado en Ferrero, Mariano. "La globalización en acción: regionalismo y paradiplomacia en Argentina y el Cono Sur Latinoamericano", VII Congreso Nacional de Ciencia Política, Sociedad Argentina de Análisis Político, noviembre 2005.

Dávila, C., Schiavon, J.A., \& Velázquez, R. (2008). Diplomacia local. Las relaciones internacionales de las entidades federativas mexicanas. México: Universidad Nacional Autónoma de México.

Duchacek, I. (1984). The international Dimension of Subnational SelfGovernment, Publius: The Journal of Federalism, 14, 5-31. 
Duchacek, I. (1986). The territorial dimension of politics: within, among and across nations. New York: Greenwood Press.

Duchacek, I. (1990). Perforated Sovereignties: Towards a Typology of New Actors in International Relations”. En H. Michelmann \& P. Soldatos. Federalism and International Relations. The Role of Subnational Units, (pp.133). Oxford: Clarendon Press.

Duchacek, I., Latouche, D., \& Stevenson, G. (Eds.) (1988). Perforated Sovereignties and International Relations: Trans-Sovereign Contacts of Subnational Governments. Nueva York: Greenwood Press.

Giménez, C., \& Malgesini, G. (2000). Guía de conceptos sobre migraciones, racismo e interculturalidad. Madrid: Los Libros de la Catarata.

Halliday, F. (2002). Las Relaciones Internacionales en un mundo en transformación. Madrid: Editorial Catarata.

Hasan Siddiqi, S. (2003). Real politics: a new approach. Pakistán: Ferozsons. Hocking, B. (2000). Vigilando la frontera: globalización, localización y capacidad de actuación de los gobiernos no centrales. En F. Aldecoa \& M. Keating, Paradiplomacia: Las relaciones internacionales de las regiones, ( $p$. 12-35). Madrid: Editorial Marcial Pons.

Iglesias, V. \& Zubelzú, G. (2008). Las provincias argentinas en el escenario internacional. Desafíos y obstáculos de un sistema federal. Buenos Aires: Programa de Naciones Unidas para el Desarrollo (PNUD).

Institución de Relaciones Internacionales Universidad de Viña del Mar. (2005). La inserción internacional de las regiones y los municipios. Lecturas sobre gestión de cooperación descentralizada. Viña del Mar: Universidad de Viña del Mar.

Kincaid, J. (2003). Foreign Relations of Sub-national Units. Constituent diplomacy in Federal Systems. En R. Blindenbacher \& A. Koller. Federalism in a Changing World. Learning from Each Other, (pp. 74-96). Quebec: McGill-Queen's University Press.

Klotz, A., \& Lynch, C. M. (2015). Strategies for Research in Constructivist International Relations. Nueva York: Routledge.

Kuznetzov, A. (2015). Theory and Practice of Paradiplomacy. Subnational governments in International Affairs. Nueva York: Routledge.

Senhoras, E. M. (2009). Geopolítica da paradiplomacia subnacional: Um estudo sobre a extroversão internacional dos municípios da rede de mercocidades. Anales del XII Encuentro de Geógrafos de América Latina (EGAL), Universidad de la República, Montevideo. 
Mateos, R. (2005). La recette de la paradiplomatie. Recuperado de: http:// www.cafebabel.fr/article/11414/la-recette-de-la-paradiplomatie.html Morgenthau, H. J. (1985). Politics among nations: the struggle for power and peace. Nueva York: Knopf Editores.

Ovando Santana, C. (2013). Algunos aspectos del desarrollo de la paradiplomacia en América del Sur vistos desde el caso chileno, Trabajos y Ensayos, 16, 1-19.

Paquin, S., (2003). Paradiplomatie identitaire et diplomatie en Belgique fédérale : le cas de la Flandre, Canadian Journal of Political Science/Revue canadienne de science politique, 3(36), 203-247.

Pont Mestres, M. (1977). Realismo político. Madrid: Plaza \& Janes.

Rachik, A. (1995). Ville et pouvoirs au Maroc. Casablanca: Editions Afrique Orient.

Seara Vázquez, M. (1988). Derecho internacional público (12 ed.). México: Editorial Porrúa.

Sepúlveda, C. (1988). Derecho internacional. México: Editorial Porrúa.

Sodupe K. (1991). The European Union and Interregional Cooperation, Regional and Federal Studies, 9(1), 58-81.

Soldatos, P. (1990). An Explanatory Framework for the Study of Federated States as Foreign-policy Actors. En H. Michelmann \& P. Soldatos. Federalism and International Relations. The Role of SubnationalUnits, (pp. 35-83). Oxford: Clarendon Press.

Soldatos, P. (1993). Cascading Subnational Paradiplomacy in an Interdependent and Transnational World. En D. Brown \& E. Fry, States and Provinces in the International Political Economy, (pp. 36-82). Berkeley: California, Institute of Governmental Studies (IGS).

Sorensen, M. (ed.) (2000). Manual de derecho internacional público. México: Fondo de Cultura Económica.

Waltz, K. N. (2001). Man, the state and war: a theoretical analysis. Nueva York, Columbia Press.

Zeraoui, Z. (2009). Los procesos mundiales contemporáneos. Trillas: México.

Zeraoui, Z. (2009). Regionalización y paradiplomacia. La politica internacional de las regiones. México: Montiel y Soriana Editores. 